\title{
Conduction Failure following Spinal Cord Injury: Functional and Anatomical Changes from Acute to Chronic Stages
}

\author{
Nicholas D. James, Katalin Bartus, John Grist, David L. H. Bennett, Stephen B. McMahon, and Elizabeth J. Bradbury \\ The Wolfson Centre for Age-Related Diseases, King's College London, London SE1 1UL, United Kingdom
}

In the majority of spinal cord injuries (SCIs), some axonal projections remain intact. We examined the functional status of these surviving axons since they represent a prime therapeutic target. Using a novel electrophysiological preparation, adapted from techniques used to study primary demyelination, we quantified conduction failure across a SCI and studied conduction changes over time in adult rats with a moderate severity spinal contusion (150 kdyn; Infinite Horizon impactor). By recording antidromically activated single units from teased dorsal root filaments, we demonstrate complete conduction block in ascending dorsal column axons acutely (1-7 d) after injury, followed by a period of restored conduction over the subacute phase ( $2-4$ weeks), with no further improvements in conduction at chronic stages (3-6 months). By cooling the lesion site, additional conducting fibers could be recruited, thus revealing a population of axons that are viable but unable to conduct under normal physiological conditions. Importantly, this phenomenon is still apparent at the most chronic (6 month) time point. The time course of conduction changes corresponded with changes in behavioral function, and ultrastructural analysis of dorsal column axons revealed extensive demyelination during the period of conduction block, followed by progressive remyelination. A proportion of dorsal column axons remained chronically demyelinated, suggesting that these are the axons recruited with the cooling paradigm. Thus, using a clinically relevant SCI model, we have identified a population of axons present at chronic injury stages that are intact but fail to conduct and are therefore a prime target for therapeutic strategies to restore function.

\section{Introduction}

Spinal cord injury (SCI) can lead to severe and permanent deficits in motor, sensory, and autonomic function. There is now a wealth of experimental studies showing improved function following various therapeutic interventions, such as targeting inhibitory myelin (Cafferty et al., 2010; Zorner and Schwab, 2010) and chondroitin sulfate proteoglycans (CSPGs) (Bradbury et al., 2002; Alilain et al., 2011; Bradbury and Carter, 2011) or by growth-promoting strategies (Alto et al., 2009) and targeting microtubule dynamics (Hellal et al., 2011). As many promising treatments are now progressing toward clinical trials, it is important for experimental studies to focus on clinically relevant injury models. The majority of human injuries involve contusion of the spinal cord (Norenberg et al., 2004), and rodent contusion models, particularly the rat, bear a striking similarity to human SCI pathology (Metz et al., 2000; Scheff et al., 2003).

While many of the pathological processes that occur following contusion injury have been well documented, a key goal in SCI

\footnotetext{
Received Aug. 22, 2011; revised Sept. 27, 2011; accepted Nov. 7, 2011.

Author contributions: D.L.H.B., S.B.M., and E.J.B. designed research; N.D.J., K.B., and J.G. performed research; N.D.J. and K.B. analyzed data; N.D.J. and E.J.B. wrote the paper.

This work was supported by the United Kingdom Medical Research Council, the International Spinal Research Trust, the International Foundation for Research in Paraplegia, the Darwin Trust of Edinburgh, and the Wellcome Trust. We thank Tom Sears for advice on electrophysiological preparations, Meirion Davies and Peter Sadeghi for help with preliminary data, Florence Fricker and Charlotte Swain for assistance with EM and data analysis, and Tim Boucher for critical evaluation of this manuscript.

The authors declare no competing financial interests.

Correspondence should be addressed to Elizabeth J. Bradbury, King's College London, Regeneration Group, The Wolfson Centre for Age-Related Diseases, The Wolfson Wing, Hodgkin Building, Guy's Campus, London Bridge, London SE1 1UL, UK. E-mail: elizabeth.bradbury@kcl.ac.uk.

DOI:10.1523/JNEUROSCI.4306-11.2011

Copyright $\odot 2011$ the authors $\quad 0270-6474 / 11 / 3118543-13 \$ 15.00 / 0$
}

research is to understand mechanisms that underlie functional changes. Electrophysiological properties of axonal projections in the contused spinal cord have previously been assessed using a variety of techniques (Metz et al., 2000; Hains et al., 2004; Beaumont et al., 2006; Hubscher and Johnson, 2006). However, as yet, the conduction properties of individual fibers that survive in the spared white matter following spinal contusion have not been studied. There is a paucity of information regarding the functional status of these surviving axons, such as whether they stabilize within the acute postinjury phase, whether there is chronic conduction block and, crucially, whether function can be restored to these axons in a chronic injury. Furthermore, although it is well known that demyelination of spared axons in the surrounding regions of a SCI occurs (Guest et al., 2005; Totoiu and Keirstead, 2005), there remains controversy over whether significant demyelination persists into chronic stages of SCI and whether this is a major contributor to loss of function (Kakulas, 1999; Lasiene et al., 2008; Salazar et al., 2010).

Here, we perform detailed functional and anatomical assessments to address these issues. By adapting techniques used to study primary demyelination, but not previously applied to SCI, we assess the temporal pattern of conduction failure and changes in the conduction properties of individual fibers following spinal contusion in adult rats over an acute to chronic time course (from $1 \mathrm{~d}$ to 6 months). We correlate changes in conduction with quantitative electron microscopy plus histological and behavioral analyses to elucidate potential mechanisms for the observed postinjury functional changes. We demonstrate complete conduction block of ascending dorsal column axons observed acutely after injury, followed by a period of improved conduction that 


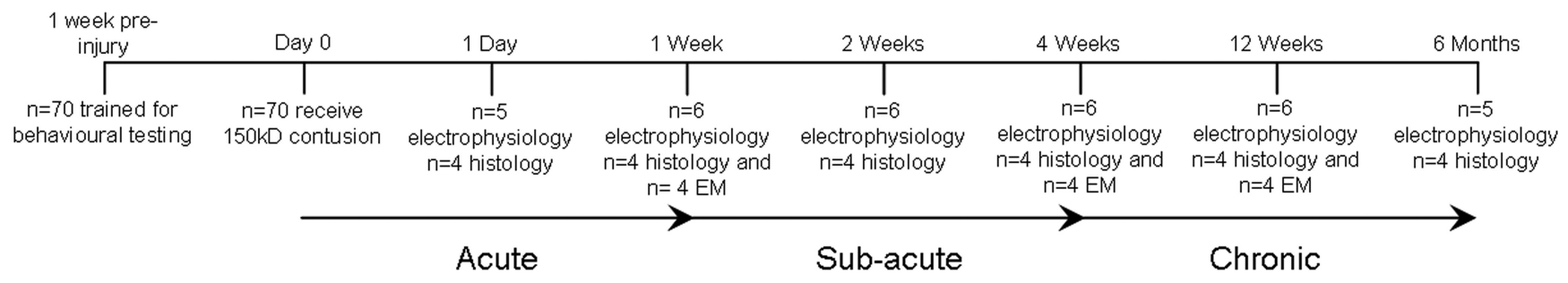

Figure 1. Timeline outlining experimental design. At a number of postinjury time points, spanning acute to chronic stages of spinal cord injury, animals were removed for terminal electrophysiological experiments, for histological assessments, or for EM. Behavioral testing was performed on all remaining animals throughout the study. This allowed a detailed assessment of functional changes as well as pathological and morphological processes that occur over time following contusion injury.

plateaus and remains stable into the chronic stages of injury. Importantly, by cooling the lesion site, we show enhanced conduction across the contusion injury, even in a chronic SCI. Thus, we have documented the time course over which viable but initially nonconducting axons regain a useful functionality and reveal a population of surviving axons that remain chronically unable to conduct under normal physiological conditions and that represent an important population to target therapeutically.

\section{Materials and Methods}

\section{Animals}

Adult female Sprague Dawley rats $(n=70 ; 200-220$ g; Harlan Laboratories) were used (housed under a $12 \mathrm{~h}$ light/dark cycle with ad libitum access to food and water). At various postinjury time points, animals were removed for electrophysiological, histological, or morphological studies $(n=5 / 6$ per time point for electrophysiology; $n=4$ per time point for histology; $n=4$ per time point for EM; Fig. 1). Behavioral testing was performed throughout the study such that all animals $(n=$ 70) were included in the first time point with fewer animals at each subsequent time point as they were assigned to the other outcome measures, so that by the final testing weeks (12 weeks and 6 months) there were $n=23$ and $n=9$, respectively. All experimental procedures were performed in accordance with the United Kingdom Animals (Surgical Procedures) Act 1996.

\section{Contusion injury surgery and postoperative care}

Animals were anesthetized using a mixture of ketamine $(60 \mathrm{mg} / \mathrm{kg})$ and medetomidine $(0.25 \mathrm{mg} / \mathrm{kg}$; administered intraperitoneally), their backs were shaved and cleansed with iodine, and core temperature was maintained close to $37^{\circ} \mathrm{C}$ using a self-regulating heated blanket. Single doses of $0.05 \mathrm{mg} / \mathrm{kg}$ buprenorphine and $5 \mathrm{mg} / \mathrm{kg}$ carprofen were given subcutaneously at the time of induction and the morning after surgery. Laminectomies were performed at vertebral level T10, the vertebral column was stabilized using Adson forceps, and the impactor probe was positioned 2-4 $\mathrm{mm}$ above the spinal cord. An impact force of $150 \mathrm{kdyn}$ was delivered to the exposed spinal cord through the intact dura with an Infinite Horizon impactor (Precision Systems Instrumentation) to create a moderate severity contusion injury. This severity was chosen since pilot studies (data not shown) revealed that more severe (200 kdyn) injuries did not spare enough dorsal column white matter to perform electrophysiological assessments in the ascending dorsal column projection and more mild (100 kdyn) injuries spared too much of the dorsal columns, making it difficult to observe any degree of change in conduction over time. This severity also mimics the $>50 \%$ of human injuries that are "incomplete" (i.e., where some white matter tissue is spared, which contains uninjured axonal projections, thus making this model very relevant to the clinical situation). Overlying muscle and skin were sutured, anesthesia was reversed using atipamezole hydrochloride $(1 \mathrm{mg} / \mathrm{kg}$ administered intraperitoneally), and animals recovered in cages placed on a heated blanket overnight. Saline $(3-5 \mathrm{ml})$ and enrofloxacin $(5 \mathrm{mg} / \mathrm{kg})$ were given subcutaneously daily for 3 and $7 \mathrm{~d}$, respectively, after injury. Bladders were manually expressed twice daily until reflexive emptying returned (typically $6-9 \mathrm{~d}$ after injury).

\section{Behavioral assessment}

Basso, Beattie, and Bresnahan locomotor scoring. Open field hindlimb locomotor function was assessed using the Basso, Beattie, and Bresnahan (BBB) locomotor rating scale (Basso et al., 1995). Briefly, this involved placing the animal in a circular open field (diameter of $1 \mathrm{~m}$ ) and assessing both hindlimbs during locomotion (over a 4 min session) for individual joint movements, paw placement, weight support, consistency of stepping and hindlimb/forelimb coordination, level of toe clearance during stepping, and overall trunk stability. Scores were calculated according to the 22 point $(0-21) \mathrm{BBB}$ scale for each hindlimb and averaged to give each animal an overall score. Testing was performed on days 1, 3, 5, and 7 after injury and weekly thereafter for 12 weeks, with a final assessment at 6 months.

Horizontal ladder. Animals were trained daily for 1 week before injury to run across a 1-m-long horizontal ladder with unevenly spaced rungs. On the final day of training, animals were recorded using a Sony DCRSX30E Handycam. Recordings were later analyzed in slow motion, allowing quantification of the total number of hindlimb footslips and misses during the course of three runs across the ladder, giving each animal a baseline score. Any form of miss or footslip that resulted in the entire paw going below the level of the rung was counted as a score of 1 . This testing procedure and analysis was then repeated once a week after injury for 12 weeks, beginning at day 7, with a final assessment at 6 months.

\section{Electrophysiological studies}

Measuring percentage conduction through the lesion and conduction velocity. Electrophysiological assessments were performed on the ascending dorsal column projection, a major spinal pathway that projects the length of the dorsal columns in the gracile fasciculus, conveying sensory information about hindlimb and hindpaw placement. Terminal electrophysiological assessments were performed at $1 \mathrm{~d}, 1$ week, 2 week, 4 week, 12 week, and 6 month postinjury time points, to assess changes in conduction across a contusion injury over acute to chronic stages of injury. For the duration of each electrophysiological experiment animals were deeply anesthetized with urethane $(1.25 \mathrm{~g} / \mathrm{kg}$; administered intraperitoneally), and depth of anesthesia was regularly assessed by monitoring pedal withdrawal reflexes and respiratory rate. Core temperature was maintained close to $37^{\circ} \mathrm{C}$ using a self-regulating heated blanket. A laminectomy was performed to remove the dorsal portions of vertebrae T7L5. The dura mater was removed from the spinal cord, exposed nervous tissue was covered with mineral oil, and silver wire stimulating electrodes were placed over the midline $\sim 5 \mathrm{~mm}$ rostral and caudal to the lesion site. Tiny filaments were teased from dorsal roots L3-S1 (both left- and righthand sides) and individually mounted on silver wire recording electrodes, allowing for the recording and quantification of single units (activity of single nerve fibers) from each of these filaments while stimulating either above or below the lesion site (Fig. 2). First, the number of single units present in each filament was counted while stimulating caudal to the lesion (5-10 single units were normally present per filament); this was then repeated while stimulating rostral to the lesion to calculate the percentage of nerve fibers capable of conducting through the lesion. In all electrophysiological experiments, the dorsal columns were stimulated using $0.2 \mathrm{~ms}$ duration square wave pulses at a frequency of $1 \mathrm{~Hz}$ and 


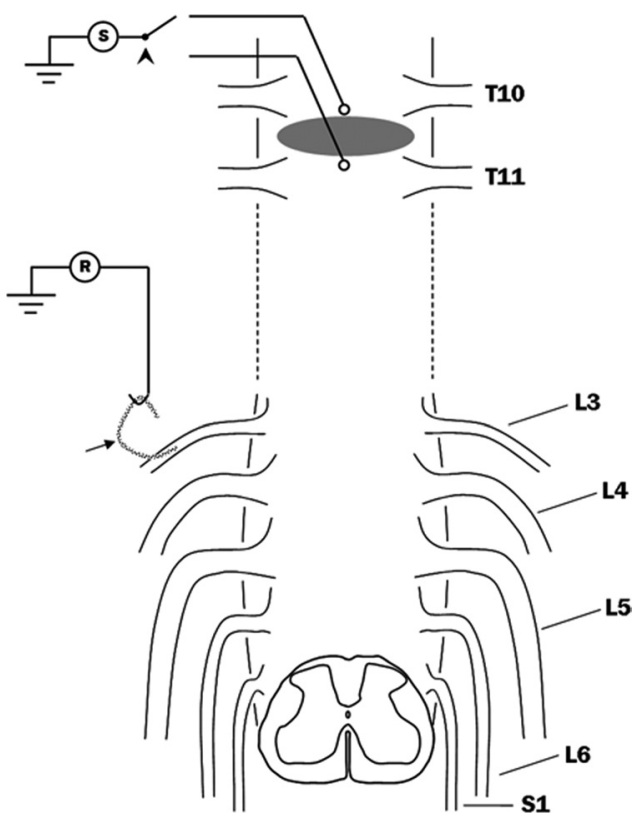

Figure 2. Schematic diagram illustrating the protocol used for assessing conduction failure in the ascending dorsal column pathway following a spinal contusion injury. Teased dorsal root filaments (arrow) were recorded from both sides of the spinal cord from dorsal roots L3-S1 while first stimulating below the injury site (gray oval), and then using a switch (arrowhead) to stimulate above the injury site, allowing a quantitative measure of the percentage of fibers capable of conducting across a contusion injury and assessment of changes in conduction from acute to chronic injury stages. S, Stimulating electrode; $R$, recording electrode.

an incrementally increasing intensity $(0-800 \mu \mathrm{A})$. During recording sessions, stimulation was continuous and lasted until all units in the filament being recorded from had been identified. At the end of each experiment, measurements were made of interelectrode distances to allow for the calculation of conduction velocities. Conduction velocities were calculated for each single unit recorded and then averaged for each animal (mean \pm SEM values were then calculated for each time point).

Cooling of the lesion. In a model of primary demyelination in the rat, it has previously been shown that cooling of the demyelinated region restored conduction in some previously nonconducting fibers (Bostock et al., 1978; Smith et al., 2000). To test the hypothesis that, following SCI, there may be some fibers that are spared but unable to conduct across the injury, we cooled the lesion site using cold mineral oil and noted the addition of any extra single units that were recruited due to this cooling while stimulating above the lesion. Cold mineral oil (in $20 \mathrm{ml}$ plastic syringes that had been stored at $-4^{\circ} \mathrm{C}$ ) was carefully applied around the lesion site to progressively cool the injury. Temperature was closely monitored using a temperature probe placed adjacent to the lesion site and connected to a tele-thermometer unit (Yellow Springs Instrument). Temperature was gradually decreased by applying small volumes of cold mineral oil at a time and a note was made of the temperature at which any additional units were added. This preparation was continued until the temperature had cooled from 37 to $15^{\circ} \mathrm{C}$. The final three to four filaments that had been recorded from in each animal were tested for any effect of cooling in all electrophysiological experiments.

Behavioral and electrophysiological correlations. Correlations of behavioral and electrophysiological data were performed on individual animals in the study $[n=4$ animals per time point that underwent electrophysiological assessments (i.e., $n=24$ for BBB tested animals and $n=20$ for ladder tested animals since they did not perform the ladder test at the $1 \mathrm{~d}$ postinjury time point)]. Percentage conduction values of individual animals were plotted against their behavioral scores (for BBB values and footslip errors) and correlation coefficients were calculated using Pearson's correlation test.

\section{Histology}

Tissue preparation. Animals were deeply anesthetized with sodium pentobarbital (Euthatal; $80 \mathrm{mg} / \mathrm{kg}$, i.p.) and transcardially perfused with $0.9 \%$ saline followed by $4 \%$ paraformaldehyde (PFA) in 0.1 м phosphate buffer $(\mathrm{PB})$. Immediately after perfusion, a section of spinal cord was removed $(\sim 10 \mathrm{~mm})$ with the lesion epicenter located centrally; this was then postfixed in $4 \%$ PFA in $0.1 \mathrm{M} \mathrm{PB}$ for $2 \mathrm{~h}$ at $4^{\circ} \mathrm{C}$ before being cryoprotected in $20 \%$ sucrose in $0.1 \mathrm{M} \mathrm{PB}$ for $48 \mathrm{~h}$. The tissue was then embedded in OCT and frozen before being cut into serial $20-\mu \mathrm{m}$-thick transverse sections using a cryostat. Sections were mounted on a series of 10 positively charged slides, such that the adjacent sections mounted on each slide represented regions spaced $200 \mu \mathrm{m}$ apart.

Immunohistochemistry. Sections were double stained for glial fibrillary acidic protein (GFAP) (a marker of reactive astrocytes) and NeuN (a neuronal cell body marker), or neurofilament 200 (NF200) (an axonal marker) and protein zero $\left(\mathrm{P}_{0}\right)$ (a peripheral myelin marker). Briefly, after blocking with $10 \%$ donkey serum in PBS containing $0.2 \%$ Triton X-100 and $0.1 \%$ sodium azide (PBST azide) for $1 \mathrm{~h}$ at room temperature (RT), the sections were incubated in PBST azide containing polyclonal rabbit anti-GFAP (1:2000; Dako) and monoclonal mouse anti-NeuN (1:500; Millipore), or monoclonal mouse anti-NF200 (clone N52; 1:400; SigmaAldrich) and polyclonal chicken anti- $\mathrm{P}_{0}$ (1:1000; Thermo Fisher Scientific) overnight at RT. After four washes of $5 \mathrm{~min}$ in PBS, sections were incubated in PBST azide containing donkey anti-rabbit Alexa 488 (1: 1000; Invitrogen) and donkey anti-mouse Alexa 546 (1:1000; Invitrogen), or donkey anti-mouse Alexa 546 (1:1000; Invitrogen) and donkey anti-chicken Alexa 488 (1:1000; Invitrogen) for $4 \mathrm{~h}$ at RT. After four washes of $5 \mathrm{~min}$ in PBS, sections were then coverslipped with Vectashield mounting medium (Vector Laboratories). Images were taken on a Zeiss LSM 710 upright confocal microscope at the same settings and in single sessions.

Eriochrome cyanine histochemistry and tissue sparing analysis. For differentiating gray and white matter, eriochrome cyanine R histochemistry was performed on spinal cord sections using a protocol adapted from previously published methods (Rabchevsky et al., 2001; Springer et al., 2010). Sections were dehydrated in an ethanol series (5 min each), cleared in Histochoice ( $5 \mathrm{~min}$ ), rehydrated in a reverse ethanol series followed by distilled water $\left(\mathrm{dH}_{2} \mathrm{O}\right)(5 \mathrm{~min}$ each), and then left in a solution containing $0.16 \%$ eriochrome cyanine $\mathrm{R}, 0.5 \%$ sulfuric acid, and $0.4 \%$ iron chloride (in $\mathrm{dH}_{2} \mathrm{O}$ ) for 10 min to stain myelinated fibers. Following staining, sections were washed twice in $\mathrm{dH}_{2} \mathrm{O}$ (5 min each), differentiated in $0.5 \%$ ammonium hydroxide $(30 \mathrm{~s})$, and washed in $\mathrm{dH}_{2} \mathrm{O}(2 \times 5 \mathrm{~min})$. Finally, sections were dehydrated and cleared, as above, and then mounted using DPX. Sections were examined using a Zeiss Axioskop microscope, and pictures were taken of sections at 800 $\mu \mathrm{m}$ intervals throughout the lesion site at each time point ( $1 \mathrm{~d}, 1$ week, 2 weeks, 4 weeks, 12 weeks, 6 months; $n=4$ per time point) using a Zeiss AxioCam MRm. Images were analyzed using AxioVision software, which allowed the tracing of the spinal cord perimeter and the central cavity for each image captured, giving the total area for each of these measurements. The lesion epicenter was defined as the section from each animal with the largest cavity area and quantification was performed at $800 \mu \mathrm{m}$ intervals from $3.2 \mathrm{~mm}$ caudal to $3.2 \mathrm{~mm}$ rostral to the epicenter. Cavity area was calculated as a percentage of spinal cord area (i.e., area within the tracing of spinal cord perimeter) for each section. Cavity volume was then calculated over a $4.8 \mathrm{~mm}$ segment of spinal cord, with lesion epicenter located centrally (the cavity very rarely extended beyond this distance).

\section{Electron microscopy}

Animals were terminally anesthetized using sodium pentobarbital (Euthatal; $80 \mathrm{mg} / \mathrm{kg}$, i.p.) and transcardially perfused with $0.9 \%$ saline followed by $3 \%$ glutaraldehyde and $4 \%$ PFA in $0.1 \mathrm{M}$ PB. Immediately after perfusion, a section of spinal cord was removed $(\sim 20 \mathrm{~mm})$ with the lesion epicenter located centrally. Three millimeter sections were taken from the lesion epicenter and from the rostral and caudal lesion borders and postfixed in the same fixative for a minimum of $48 \mathrm{~h}$ at $4^{\circ} \mathrm{C}$. After washing in $0.1 \mathrm{M} \mathrm{PB}$, sections were osmicated, dehydrated in a graded ethanol series, and embedded in epoxy resin (TAAB Embedding Materi- 
als). One micrometer transverse semithin spinal cord sections were cut on a microtome and stained with $0.25 \%$ toluidine blue solution before being examined on a Zeiss Axioskop light microscope to determine the region of the lesion epicenter to be further processed for ultrastructural analysis. For electron microscopy (EM), blocks containing the lesion epicenter were trimmed and transverse ultrathin sections were cut on an ultramicrotome and stained with lead uranyl acetate by the Centre for Ultrastructural Imaging (King's College London, London, UK). Ultrathin sections were mounted on unsupported 100 mesh grids and were visualized on a Hitachi H7600 transmission electron microscope. For analysis, photographs of the region containing the ascending dorsal column projection from each animal were taken at a magnification of $6000 \times$. Full montages of grid squares were taken, and randomly chosen images from a given grid square were analyzed. For analysis of proportion of nonmyelinated axons with diameters $>1 \mu \mathrm{m}$, myelinated and nonmyelinated axons were analyzed from the same randomly chosen images. For analysis of axons depending on their location relative to the cavity border, a separation was made between grid squares located furthest away or closest to the cavity border. Randomly selected images from at least two grid squares, one of which was adjacent to the cavity border and one of which was bordering the dorsal edge of the spinal cord, were then analyzed and compared with respect to $G$ ratio values and nonmyelinated axons. The $G$ ratios of $\sim 500$ axons and nonmyelinated axon diameters of $\sim 200$ to 300 axons, chosen randomly, were measured per animal using AxioVision LE, release 4.2, software.

\section{Statistical analysis}

For analysis of behavioral deficits, electrophysiological percentage conduction, and tissue volume, a one-way ANOVA was used followed by Tukey's post hoc test. Conduction velocity and cavity area measurements and comparisons of myelin at differing locations were analyzed using a two-way repeated-measures (RM) ANOVA followed by Bonferroni's post hoc comparison. $G$ ratio frequency distributions were analyzed using a Kolmogorov-Smirnov test. All statistical tests were conducted using GraphPad Prism 5. All data are presented as mean \pm SEM, and $p<0.05$ was classed as significant.

\section{Results}

\section{Assessment of behavioral deficits following contusion injury}

$B B B$ locomotor rating

$\mathrm{BBB}$ scoring was performed to assess changes in gross locomotor performance following contusion injury (Fig. 3A). Before contusion surgery, all animals achieved a baseline score of 21 , indicating normal locomotion and hindlimb function. At all postinjury time points (from $1 \mathrm{~d}$ to 6 months), animals were substantially and significantly impaired compared with baseline values $(p<$ 0.001, one-way ANOVA, Tukey's post hoc). Locomotor function was most severely impaired acutely postinjury, with a mean BBB score of $3.4 \pm 0.6$ at $1 \mathrm{~d}$ after injury (representing extensive movement of two hindlimb joints or slight movement of all three hindlimb joints). Some spontaneous recovery was observed over the initial 2 week period after injury, with a mean BBB score of $10.2 \pm 0.4$ by 1 week after injury, indicating that animals were now capable of occasional weight-supported steps but that there was a complete lack of forelimb-hindlimb coordination. By 2
B

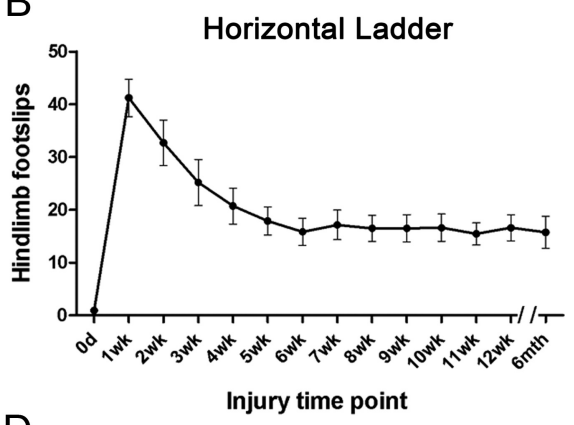

D

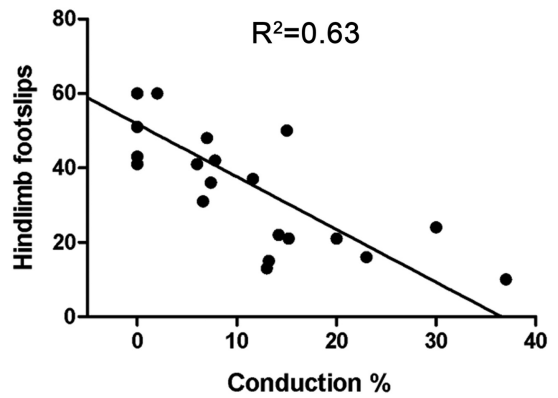

Conduction $\%$ 
A

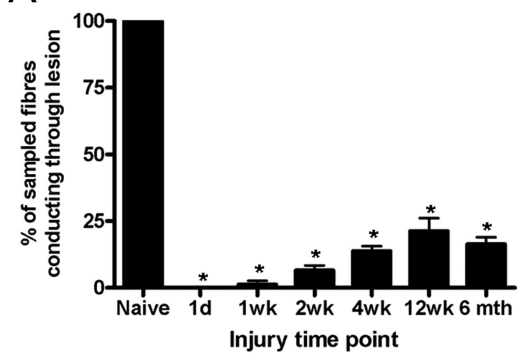

C

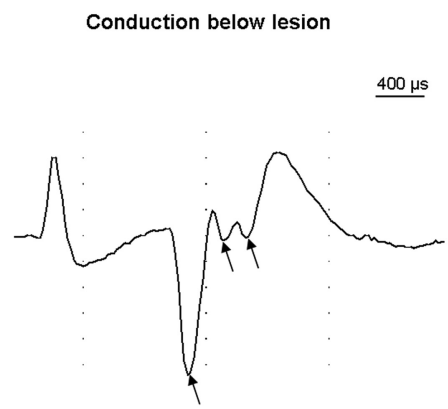

E

Effect of cooling: $37^{\circ} \mathrm{C}$

$400 \mu \mathrm{s}$

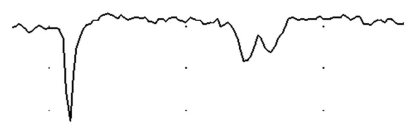

B

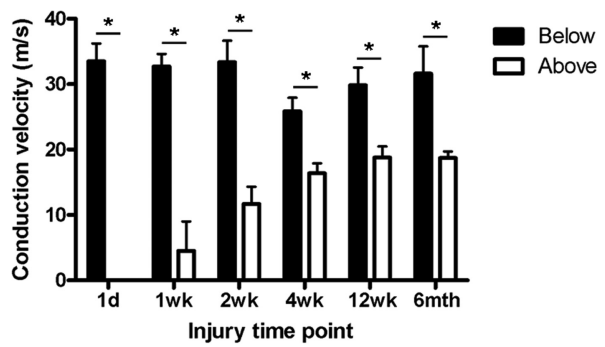

D

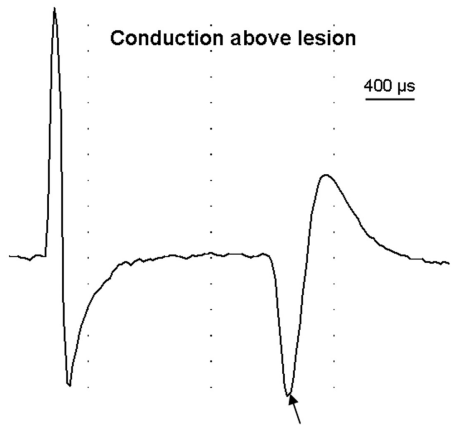

$\mathrm{F}$

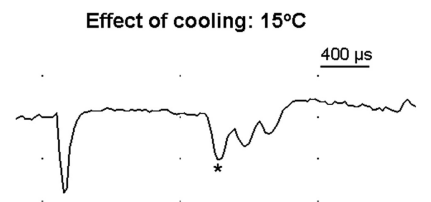

Figure 4. A $150 \mathrm{kDa}$ contusion leads to a substantial decrease in the percentage of fibers capable of conducting across a contusion injury and a slowing of their conduction velocity. $\boldsymbol{A}$, Functionality of the ascending sensory pathway is initially abolished postinjury, with a complete lack of conduction acutely $(1 \mathrm{~d})$, followed by a partial recovery of conduction over the subacute phase (with $\sim 14 \%$ of sampled fibers capable of conducting across the contusion injury at 4 weeks); no further improvements in conduction properties of ascending dorsal column axons were observed, with the functionality of the dorsal column pathway remaining severely impaired at the chronic postinjury time points $(\sim 16 \%$ of sampled fibers capable of conducting across the contusion injury at chronic stages of spinal cord injury). $\boldsymbol{B}$, Conduction velocity measurements of individual fibers capable of conducting through the lesion site (above injury recordings) revealed a significant slowing of conduction velocity at every postinjury time point, compared with the conduction velocity of fibers activated below the injury (below injury recordings), where this tract would be relatively intact (although, as with the percentage conduction measurements, there was some recovery in conduction velocity over the subacute stages of injury). Representative traces recorded while stimulating below $(\boldsymbol{C})$ and then above $(\boldsymbol{D})$ the injury site highlight the differences in conduction properties between the "intact" and injured pathway. Three single units are present while stimulating below the injury, but only one survives to conduct through the lesion. The delayed latency of the unit conducting through the lesion indicates impaired conduction velocity. Single units of activity (arrows) represent the activity of single nerve fibers. $\boldsymbol{E}$ and $\boldsymbol{F}$ show representative traces from above the injury where the cooling technique was applied. By cooling the lesion site with cold mineral oil, additional conducting fibers could be recruited at every postinjury time point (the asterisk in $\boldsymbol{F}$ highlights a single unit added following cooling), thus revealing an important population of axons that are viable but unable to conduct under normal physiological conditions. Error bars represent SEM. The asterisks in $\boldsymbol{A}$ denote significantly impaired conduction of dorsal column axons following contusion, compared with conduction properties in the uninjured (naive) spinal cord ( $p<$ 0.001 , one-way ANOVA, Tukey's post hoc); the asterisks in $\boldsymbol{B}$ denote significantly impaired conduction velocity of dorsal column axons recorded above, compared with below, the injury ( $p<0.01$, two-way RM ANOVA, Bonferroni's post hoc).

point, with footslip errors remaining at this level into chronic postinjury stages (15.92 \pm 3.1 at 6 months after injury).

Correlations between behavior and electrophysiology

Since the two behavioral tests showed a different recovery time course, we correlated the behavioral scores of individual animals with their conduction scores (see percentage conduction data below). BBB scores showed a low correlation with the percentage of conducting fibers $\left(R^{2}=0.34\right)$, such that improved conduction through the injury did not always correspond with higher BBB scores; this was particularly apparent over the subacute phase in which conduction is still improving but BBB scores have already reached a plateau (Fig. 3C). In contrast, there was a high correlation between footslip errors on the ladder task and percentage fiber con- duction $\left(R^{2}=0.63\right)$, such that improved conduction through the injury site typically corresponded with fewer footslip errors on the horizontal ladder (Fig. 3D). Thus, the horizontal ladder task may be a more sensitive test for revealing subtle changes in function in this injury model.

\section{Electrophysiological assessment of conduction failure following contusion injury \\ Conduction of the ascending dorsal column pathway}

By stimulating below the injury site, activating the ascending sensory pathway antidromically and recording from dorsal root filaments, it was possible to quantify the number of functional fibers in that particular filament. Stimulating above the injury site then allowed quantification of the percentage of those fibers surviving and still capable of conduction through the lesion (Fig. $4 A, C, D$ ). In naive animals, all single units (activity of single nerve fibers) observed while stimulating with the caudally positioned electrode were still present when stimulation was switched to the rostral electrode, indicating that both electrodes were capable of maximally activating each of the filaments tested. As with behavioral assessment of function, spinal cord contusion resulted in substantial and significantly impaired function, with a dramatic reduction in the percentage of conducting fibers observed at all postinjury time points compared with naive animals $(p<0.001$, one-way ANOVA, Tukey's post hoc; Fig. 4A). Acutely postinjury $(1 \mathrm{~d})$, there was a complete lack of conduction through the lesion site. This lack of conduction persisted at 1 week after injury in most cases, although one animal at this time point displayed a limited amount of functionality resulting in a mean level of conduction of $1.32 \pm$ $1.32 \%$. As with behavioral function, some spontaneous improvement in conduction occurred over the subacute stages of injury. By 2 weeks after injury, there were a small number of fibers capable of conducting through the lesion in all animals tested $(6.5 \pm 1.87 \%)$, which continued to increase until 4 weeks after injury (13.73 \pm $1.78 \%)$; this marked a significant improvement compared with the level of conduction observed at 1 week after injury ( $p<0.05$, oneway ANOVA, Tukey's post hoc). There were no further significant improvements in conduction at later time points, with the percentage of sampled fibers capable of conducting across a contusion injury remaining at this level into chronic postinjury stages (16.36 \pm $2.57 \%$ at 6 months after injury).

Conduction velocities of surviving fibers

One advantage of recording from a direct pathway (i.e., no synapses involved) was that it allowed an accurate calculation of the 
conduction velocity of the individual fibers recorded from. At every time point assessed, there was a significant slowing in the conduction velocity of the fibers that remained capable of conduction through the lesion site when compared with the conduction velocity of fibers activated when stimulating caudal to the lesion (Fig. $4 B-D ; p<0.05$, two-way RM ANOVA, Bonferroni's post hoc). This suggested that, although these fibers were capable of conduction, they were not functioning in an entirely normal manner. As with percentage conduction, conduction velocity of the spared fibers significantly improved over the first 4 weeks after injury when compared with conduction velocities at 1 week after injury $(4.51 \mathrm{~m} / \mathrm{s}$ compared with $16.38 \mathrm{~m} / \mathrm{s}$ at 1 and 4 weeks, respectively; $p<0.001$, two-way RM ANOVA, Bonferroni's post hoc). There were no further increases in conduction velocity of fibers conducting through the lesion at chronic (12 week, 6 month) postinjury stages.

\section{Enhanced conduction following cooling} Cooling of nerve fibers can lower their threshold for activation due to differences in the temperature sensitivities of sodium and potassium channels responsible for action potential propagation. Previously, this technique has been used to restore conduction across regions of focal demyelination (Smith et al., 2000); here, it was used to test the hypothesis that there may be some fibers that had survived through the lesion site but that were not capable of conduction under normal physiological conditions. While the use of this technique had no effect on the number of fibers conducting in naive (uninjured) spinal cords, there were numerous examples of at least one single unit being added to the recording from a dorsal root filament following cooling of the lesion site at all postinjury time points, excluding $1 \mathrm{~d}$ after injury (Fig. $4 E, F)$, with the addition of at least one single unit observed in 10 of 22 filaments at 1 week after injury, 4 of 20 at 2 weeks, 7 of 26 at 4 weeks, 6 of 25 at 12 weeks, and 5 of 28 at 6 months. Thus, there were viable, nonconducting axons present in $\sim 20 \%$ of the filaments tested at all postinjury time points other than $1 \mathrm{~d}$ after injury.

\section{Ultrastructural assessment of demyelination and} remyelination following contusion injury

Electron microscopy

The dorsal column region containing the fasiculus gracilis was chosen for electron-microscopic analysis as it contained the axons from which electrophysiological recordings were performed (see Fig. 6A$D$ ). Dorsal column axons from naive (uninjured) spinal cords were
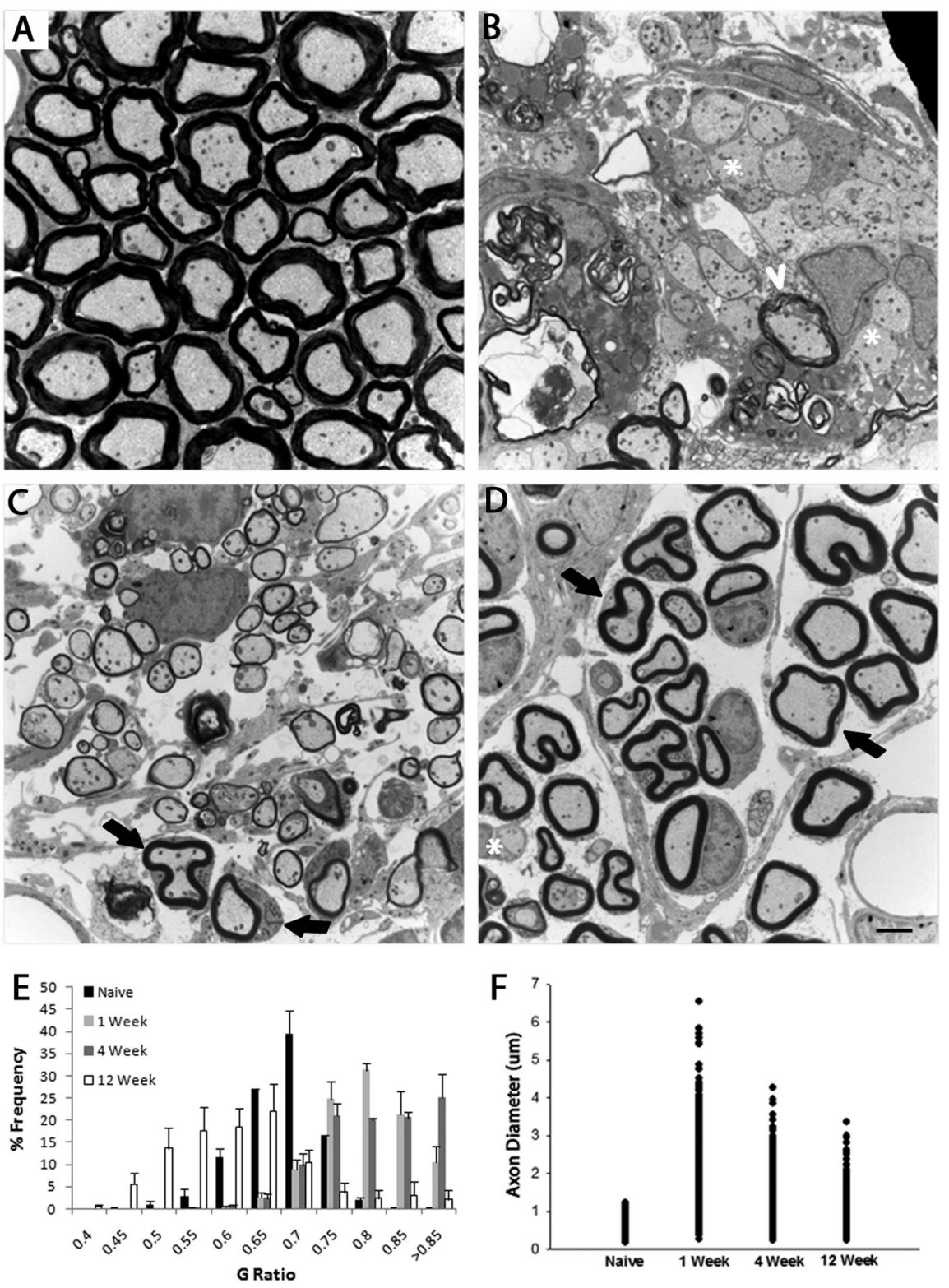

Figure 5. Time course of demyelination and subsequent remyelination of dorsal column axons at the contusion lesion epicenter. $\boldsymbol{A}$, Electron micrograph of dorsal column axons from an uninjured (naive) spinal cord illustrating healthy myelin sheaths. $\boldsymbol{B}$, At 1 week after injury, extensive demyelination is apparent (asterisks) and remaining myelin appears unhealthy as it becomes less compact and often appears to be unraveling (white arrowhead). C, By 4 weeks after injury, some remyelination is apparent and both nonmyelinated and thinly remyelinated axons are found throughout the dorsal columns. Some axons associated with Schwann cells are also apparent (black arrows). $\boldsymbol{D}$, Remyelination progresses and at 12 weeks after injury many axons have myelin sheaths that are thick and compact in appearance and are often associated with Schwann cells (black arrows). $\boldsymbol{E}$, G ratio frequency distributions show a significant shift toward high proportions of thinly myelinated axons at 1 and 4 weeks after injury compared with naive (uninjured) dorsal column axons ( $p<0.0001$, KolmogorovSmirnov test), with this shift reversed by 12 weeks after injury. $F$, Graphic plot displaying numbers and diameter of nonmyelinated axons measured at each time point shows that significant populations of large-diameter axons $(>1 \mu \mathrm{m})$ that lacked myelin sheaths were present following contusion injury at all postinjury time points, compared with uninjured spinal cords where only axons with a diameter of $\leq 1 \mu \mathrm{m}$ were nonmyelinated ( $p<0.001$, one-way ANOVA, Tukey's post hoc test). Scale bar, $2 \mu \mathrm{m}$.

normal in appearance (Fig. 5A), while numerous axons either lacking myelin sheaths or with disrupted and unhealthy myelin were observed at 1 week after injury (Fig. 5B). By 4 weeks after injury, many axons appeared to be very thinly myelinated and often remyelination had been performed by Schwann cells, identified by the presence of a basal lamina and the characteristic features of Schwann cell nuclei (Fig. $5 C$ ), suggesting that remyelination was taking place and was often mediated by Schwann cells. As the injury progressed into chronic stages (12 weeks), many axons now had dense, healthy 

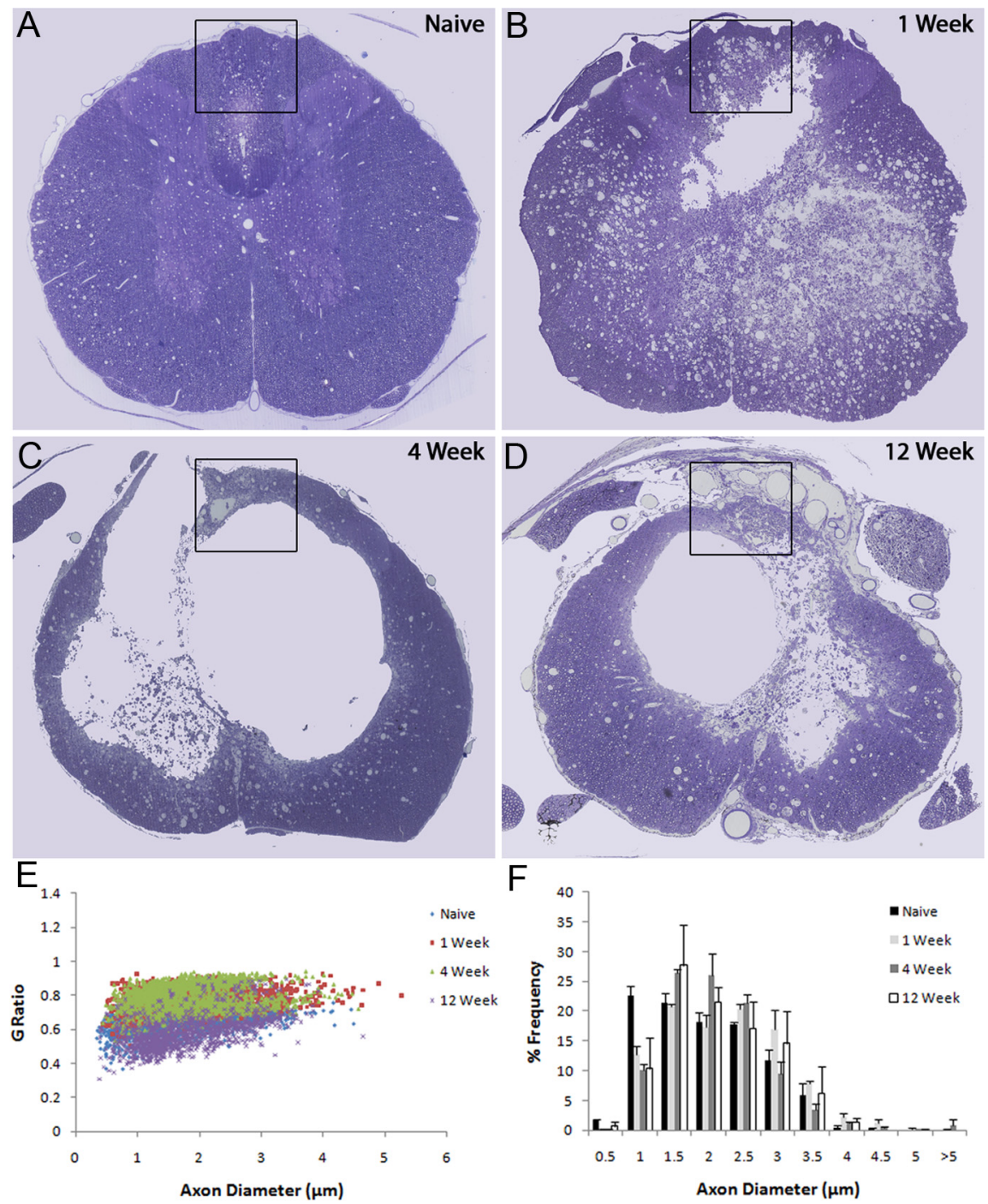

Figure 6. Transverse semithin spinal cord sections, stained for toluidine blue, taken from an uninjured (naive) spinal cord $(\boldsymbol{A})$ or from the lesion epicenter at 1 week $(\boldsymbol{B}), 4$ weeks $(\boldsymbol{C})$, and 12 weeks $(\boldsymbol{D})$ after contusion injury. The boxed areas indicate the regions processed for electron microscopy, containing the ascending dorsal column axons, and correspond to the electron micrographs in Figure $5 A-D$. Shown are a scatter plot $(\boldsymbol{E})$ and frequency distribution $(\boldsymbol{F})$ of diameters of myelinated axons. No significant changes in axon calibre were found at any of the postinjury time points assessed.

myelin sheaths and, again, this was often associated with Schwann cells (Fig. 5D). There was a significant increase in the mean $G$ ratio at 1 week (mean $G$ ratio $=0.77 \pm 0.012$ ) and 4 weeks (mean $G$ ratio $=$ $0.79 \pm 0.01$ ), compared with axons in uninjured spinal cords (mean $G$ ratio $=0.64 \pm 0.023 ; p<0.02$, one-way ANOVA, Tukey's post $h o c)$, with a significant shift in $G$ ratio frequency distribution ( $p<$ 0.0001 , Kolmogorov-Smirnov test; Fig. $5 E$ ). This shift had reversed by 12 weeks after injury, indicating that most axons had comparable, or thicker, myelin sheaths than those observed in uninjured spinal cord (Fig. 5E), which presumably is a consequence of Schwann cell remyelination. No significant changes in axon calibre of myelinated axons were found at any of the time points assessed (Fig. 6E,F). Additionally, while in uninjured spinal cords only axons with a diameter of $\leq 1 \mu \mathrm{m}$ were nonmyelinated, significant populations of nonmyelinated large-diameter axons $(>1 \mu \mathrm{m})$ were present following contusion injury at all postinjury time points $(p<0.001$, oneway ANOVA, Tukey's post hoc test; Fig. $5 F$ ). Thus, at all postinjury time points there remained a proportion of axons that were demyelinated following injury and had not been remyelinated.
Analysis was performed to determine whether there were any differences in myelin sheath thickness and/or proportion of large-diameter $(>1 \mu \mathrm{m})$ nonmyelinated axons depending on the location of the axons relative to the cavity border. Dorsal column axons located furthest away from the cavity border were compared with axons closest to the cavity (Fig. $7 F$ ). G ratio quantification revealed no difference between these two locations in the uninjured spinal cord ( $p>0.05$, two-way ANOVA; Fig. $7 A)$ or at 12 weeks after injury $(p>$ 0.05, two-way ANOVA; Fig. 7D). In contrast, at 1 week (Fig. $7 B$ ) and 4 weeks (Fig. 7C) after injury, there was a significant increase in the mean $G$ ratio of axons close to the cavity border compared with axons located in the most dorsal regions of the dorsal columns (mean $G$ ratio for 1 week after injury $=0.80 \pm 0.01$ and $0.76 \pm 0.02$ for closest to and furthest away from cavity, respectively; mean $G$ ratio for 4 weeks after injury $=0.82 \pm 0.01$ and $0.76 \pm 0.01$ for closest to and furthest away from cavity, respectively; $p<0.05, p<0.001$ for frequency distribution dorsal vs ventral location at 1 and 4 weeks after injury, respectively; two-way ANOVA, Bonferroni's post hoc). Similarly, there were significantly more large-diameter $(>1 \mu \mathrm{m})$ nonmyelinated axons observed closest to the cavity border at 1 and 4 weeks after injury $(p<0.05$ and $p<0.001$ for dorsal vs ventral location at 1 and 4 weeks, respectively; two-way ANOVA, Bonferroni's post hoc; Fig. 7E). A similar trend was apparent at 12 weeks after injury but did not reach statistical significance.

\section{Schwannosis in the dorsal columns}

following contusion injury

Schwannosis is frequently observed in the chronically injured human spinal cord (Bruce et al., 2000; Guest et al., 2005) where, as a result of Schwann cell invasion and/or proliferation, central axons become associated with peripheral myelin. Colocalization of the neurofilament marker NF200 with the Schwann cell marker $\mathrm{P}_{0}$ at the lesion epicenter revealed positive staining for peripheral myelin around numerous dorsal column axons at 12 weeks following contusion injury (Fig. 8). NF200 and $\mathrm{P}_{0}$ colocalization in the dorsal columns was also apparent at the 4 week postinjury time point, but not at earlier (1 week) stages postinjury (Fig. 9). These findings are in agreement with the ultrastructural observations and indicate that, as the injury progresses into the chronic stages, Schwann cells present in the dorsal columns following contusion injury form myelin around demyelinated axons.

\section{Histological assessment of lesion pathology following contusion injury \\ Cavity formation, reactive gliosis, and neuronal cell loss}

Costaining of transverse sections with NeuN (a neuronal cell marker) and GFAP (an astrocyte marker) revealed a number of progressive pathological changes that occur over time following 
contusion injury. At the lesion epicenter, these were characterized by a loss of neuronal cell bodies, mass destruction of spinal cord gray matter, increasing cavity formation, and reactive gliosis (Fig. 10A-F). Acutely (1 d) postinjury, marked necrosis of the gray matter was already apparent, with very few NeuN-positive neuronal cell bodies remaining (Fig. 10B); this progressed over the subacute (1-2 weeks; Fig. 10C,D) to chronic (4-12 weeks; Fig. 10E,F) stages, with a complete absence of NeuN-positive cell bodies from 2 weeks after injury and necrosed tissue areas giving rise to chronic central cavities. Costaining with GFAP showed a dramatic increase in reactive astrocytosis throughout the lesion epicenter at the acute stage (Fig. $10 \mathrm{~B}$ ). As the injury progressed, the reactive gliosis appeared to become more focused on the perimeter of the developing cavities, forming a dense glial scar around them (Fig. 10C-F). Differentiation of gray and white matter using eriochrome cyanine R histochemistry highlighted the extent and progression of gray and white matter loss from acute to chronic stages following contusion injury, with debris and necrosed tissue in the central core of the lesion at early postinjury stages being gradually cleared to leave behind a large central cavity, surrounded by a rim of spared white matter (Fig. 10G-L).

\section{Tissue sparing}

To quantify the extent of cavity formation at the lesion epicenter as well as the rostrocaudal spread of the injury, analysis was performed on serial sections stained with eriochrome cyanine R (Fig. 11). The lesion epicenter was classified as the tissue section in which cavity area was largest. At each time point, there was severe pathology apparent in the lesion epicenter, where mass destruction of both gray and white matter occurred; at rostrocaudal distances from the epicenter, the areas of necrosis and cavitation were predominantly confined to the dorsal columns (Fig. 11A). Quantification revealed that, at acute and subacute time points ( $1 \mathrm{~d}$ to 2 weeks), the cavity area at the lesion epicenter was $\sim 10 \%$ of the total cord area. The cavity area then dramatically increased at the later postinjury time points $(\sim 25,30$, and $40 \%$ of the total cord area at 4 weeks, 12 weeks, and 6 months after injury, respectively; Fig. $11 B$ ). At all chronic time points ( 4 week, 12 week, and 6 month), there was a significant increase in cavity area at lesion epicenter compared with all acute and subacute time points ( $1 \mathrm{~d}, 1$ week, and 2 weeks; $p<$ 0.05, two-way RM ANOVA, Bonferroni's post hoc). This is consistent with the previous observation that before 4 weeks the lesion core is filled with necrosed tissue and debris, which then gives rise to a central cavity. The rostrocaudal spread of the lesion also progressively increased over this period, with greater cavity areas observed at sites increasingly distal to the lesion epicenter over time (Fig. 11 A,B). Measurements of total cavity volume at each time point confirmed a cavity (green).

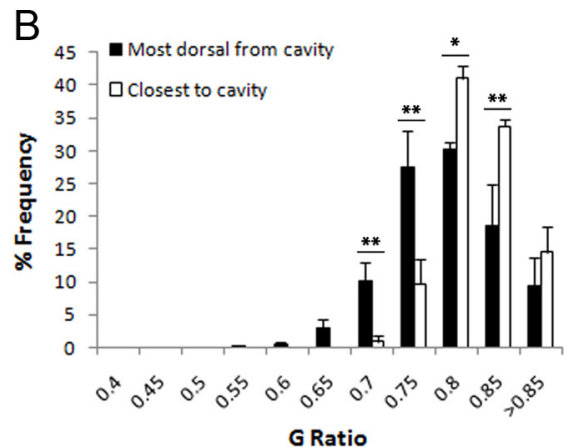

$\mathrm{D}$

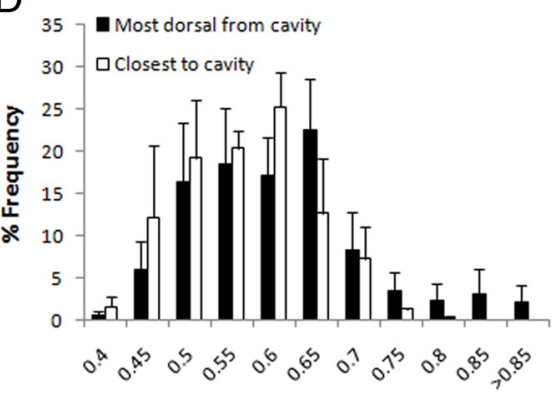

$\mathrm{F}$
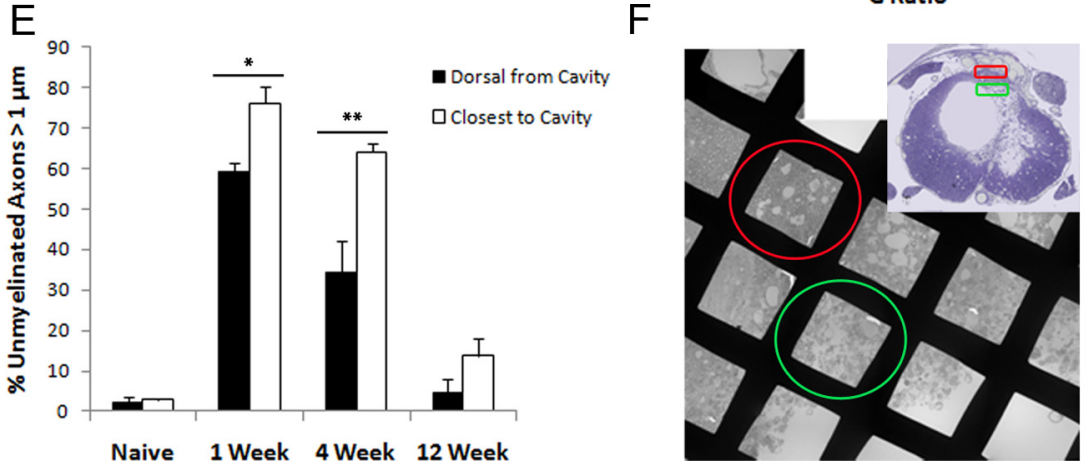

Figure 7. Comparison of the state of myelination of dorsal column axons located either furthest away from the cavity edge (dorsal from cavity) or adjacent to the cavity (closest to cavity). $A-D, G$ ratio frequency distributions reveal no differences in myelin after injury, many more thinly myelinated axons are located close to the cavity border while axons with thicker myelin location from the cavity border reveals that at 1 week and 4 weeks after injury significantly more nonmyelinated axons are found close to the cavity border $\left({ }^{*} p<0.05 ;{ }^{* *} p<0.001\right.$; two-way ANOVA Bonferroni's test). $\boldsymbol{F}$, Illustration of compared locations of axons relative to the cavity border. Axons furthest away from the cavity border (red) were compared with axons located close to the

progressive increase from subacute ( 2 weeks) to chronic (6 months) time points in the total area through the lesion that contained cavities (Fig. 11C). As with cavity area, total cavity volume at chronic time points was significantly higher than that observed at acute and subacute time points ( $p<0.01$, one-way ANOVA, Tukey's post hoc).

\section{Discussion}

This study demonstrates for the first time the temporal pattern of conduction failure of individual fibers across a contusion injury and examines changes in their conduction properties from acute to chronic stages. At different postinjury time points (from $1 \mathrm{~d}$ to 6 months), we used electrophysiological, behavioral, and anatomical assessments to document the time course over which (1) spared axons regain conduction, (2) animals regain hindlimb function, and (3) spared axons remyelinate. By antidromic recordings above and below the injury, we quantified the time 


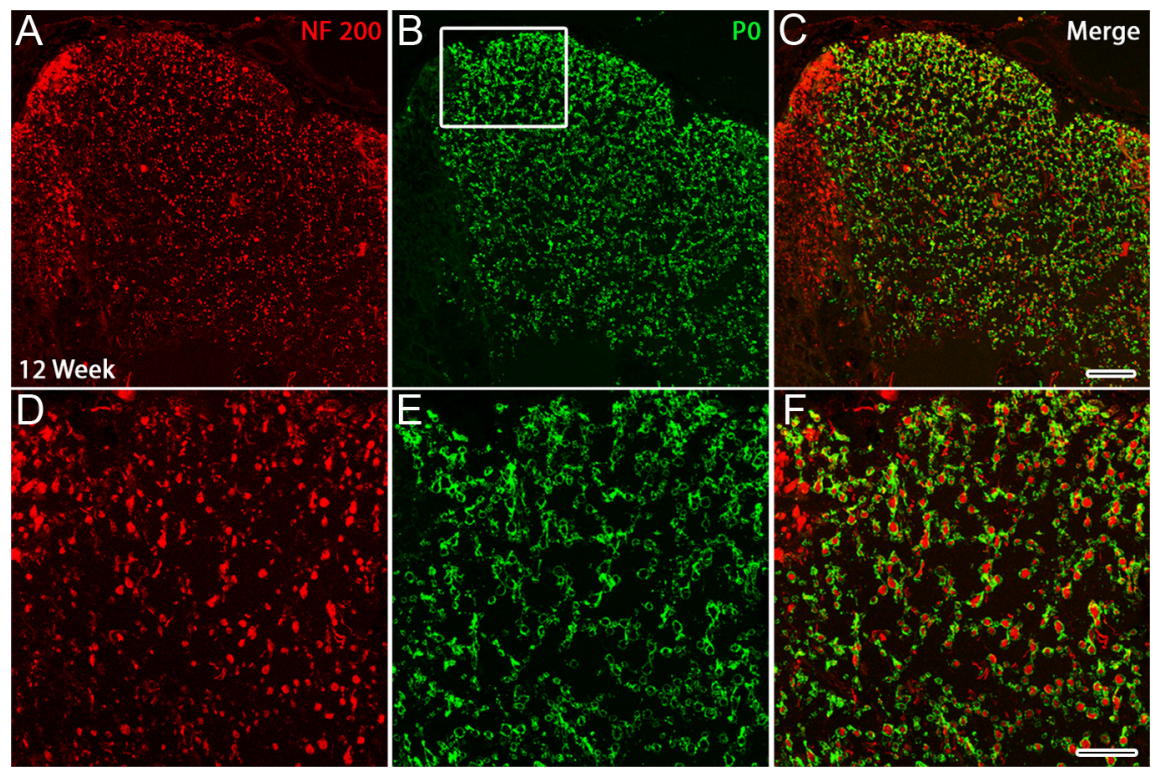

Figure 8. Remyelination of dorsal column axons at the lesion epicenter is frequently associated with Schwann cells. Costaining of axons (NF 200; red) and Schwann cell-associated myelin ( $\mathrm{P}_{0}$; green) illustrates the presence of remyelinating Schwann cells in the dorsal column of the spinal cord at the lesion epicenter at 12 weeks after injury. The bottom panels $(\boldsymbol{D}-\boldsymbol{F})$ depict the boxed area in the top panels $(\boldsymbol{A}-\boldsymbol{C})$ at higher magnification (63× oil). Scale bars: $\boldsymbol{A}-\boldsymbol{C}, 50 \mu \mathrm{m} ; \boldsymbol{D}-\boldsymbol{F}, 20 \mu \mathrm{m}$.
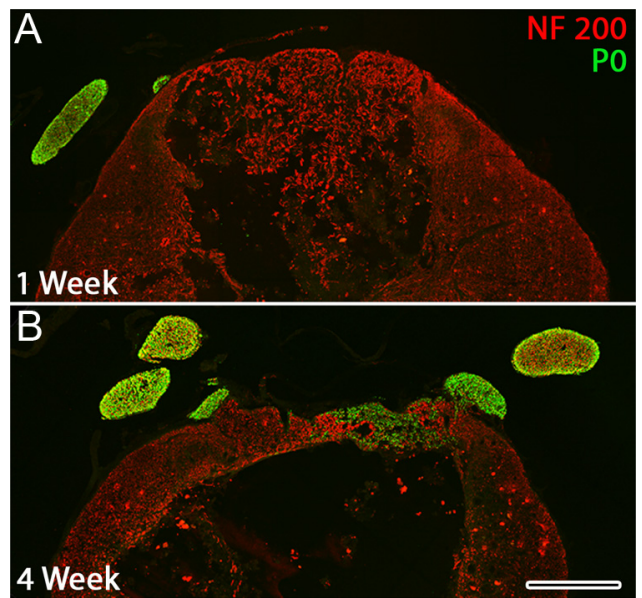

Figure 9. Costaining of axons (NF 200; red) and Schwann cell-associated myelin ( $P_{0}$; green) illustrates the presence of remyelinating Schwann cells in the dorsal columns of the spinal cord at the lesion epicenter at 4 weeks $(\boldsymbol{B})$ but not at 1 week $(\boldsymbol{A})$ after injury.

course over which viable but initially nonconducting axons regain function, with conduction restored to $\sim 15 \%$ of fibers within 4 weeks of contusion following acute postinjury conduction block. We also reveal a population of viable but nonconducting axons at chronic stages of a SCI in which conduction can be restored by cooling the injury site. EM analysis revealed the pattern of demyelination and subsequent remyelination after SCI, with a time course that corresponded to functional changes, and also revealed a population of chronically demyelinated axons, which are likely to be the population of axons that responded to cooling. Thus, these studies provide mechanistic insight into functional changes that occur following SCI and identify an important population of axons that are chronically functionally compromised but remain accessible to strategies to restore conduction.

\section{Conduction failure following spinal contusion}

The immediate loss of conduction at $1 \mathrm{~d}$ after injury is likely due to the effects of spinal shock (Atkinson and Atkinson, 1996; Smith and Jeffery, 2005), a process that is thought to be caused by a number of contributing factors such as physical damage to axons, local hemorrhage, and ischemia (Tator and Fehlings, 1991; Dumont et al., 2001). Hemorrhage can also lead to the increase of molecules that are inhibitory to conduction such as nitric oxide (NO) (Sharma et al., 1996; Winkler et al., 1998), and damage, or loss, of oligodendrocytes classically leads to demyelination and conduction block (McDonald and Sears, 1969; Shuman et al., 1997). EM analysis at 1 week after injury revealed fully demyelinated axons as well as unhealthy noncompacted myelin, suggesting that demyelination was an important contributing factor to the loss of conduction in the present study. Finally, CSPGs may also play a role in conduction failure postcontusion, since CSPGs are known to be highly upregulated at SCI sites (Jones et al., 2003; Tang et al., 2003; Iaci et al., 2007) and acute application of CSPGs can block axonal conduction in the spinal cord (Hunanyan et al., 2010).

At all time points, the small proportion of axons that conducted across the injury had significantly reduced conduction velocities. Despite significant remyelination taking place, conduction velocities remain significantly impaired at 6 months after injury. We show here that the remyelination is primarily mediated by Schwann cells, which are likely to have different properties to central myelin. Additionally, the average internodal length of newly formed myelin following some demyelinating lesions is significantly shorter than normal (Blakemore and Murray, 1981), which may also contribute to the impaired conduction velocity in the present study.

\section{Novel population of viable but nonconducting axons}

This is the first time that conduction changes in individual fibers have been assessed in vivo following spinal contusion in the adult rat. A number of previous studies have examined motor or sensory evoked potentials following contusion, often using noninvasive magnetic stimulation paradigms and/or minimally invasive needle electrodes (Khan et al., 1999; Metz et al., 2000; Cao et al., 2005; Beaumont et al., 2006; Ellingson et al., 2008), providing information regarding the effects of contusion on complete tracts in the spinal cord. Hains et al. (2004) looked in more detail at conduction of sensory axons across a contusion injury by stimulating the lumbar dorsal columns and recording compound action potentials above the contusion injury, to assess protective effects of sodium channel blockade on conduction of the dorsal column pathway 1 month after injury. In the present study, we record from individual fibers teased from dorsal root filaments to obtain detailed information on spared fibers and thus reveal important information about the integrity of the fiber, such as whether it is damaged or demyelinated. Conduction properties of sensory fibers have previously been examined in isolated spinal cord segments following compression injury (Blight, 1983; Nashmi and Fehlings, 2001) and these ex vivo tech- 
niques have been used to demonstrate the role of voltage-gated potassium channels in chronic axonal dysfunction (Nashmi et al., 2000). The present study supports previous observations on the loss and recovery of evoked potentials following thoracic and cervical contusion injury (Nashmi et al., 1997; Onifer et al., 2007) and also suggests that demyelination is a major factor in chronic conduction failure, since a key finding was that lowering the activation threshold of fibers by cooling the lesion site revealed the presence of viable nerve fibers that are unable to conduct under normal physiological conditions. Studies of primary demyelinating lesions have used a similar cooling paradigm to successfully restore conduction in some fibers (Rasminsky, 1973; Bostock et al., 1978; Smith et al., 2000), but this technique has not previously been applied to a SCI study. Cooling of the lesion prolongs action potential duration due to differences in the temperature sensitivity of sodium and potassium channels, increasing the likelihood of action potential propagation over long distances if myelination is focally impaired (Bostock et al., 1978). Thus, we provide robust evidence of a population of spared axons that remain chronically compromised and unable to conduct following SCI and, importantly, that they remain accessible to strategies to restore conduction and that this can have a significant functional effect. It will be important to study and characterize this population further, since restoring their conduction could lead to significant functional improvements, which is potentially more easily achievable than more complex regeneration-promoting strategies.

\section{Chronic demyelination following spinal contusion}

Whether demyelination persists in chronic SCIs and, if so, whether it occurs significantly enough to affect function, remains controversial in the field. Conflicting reports have appeared from both human and experimental studies, in which some reports claim little or no evidence of chronic demyelination (Kakulas, 1999; Lasiene et al., 2008; Salazar et al., 2010), while others report significant progressive chronic demyelination (Waxman, 1989; Bunge et al., 1993; Guest et al., 2005; Totoiu and Keirstead, 2005). Strategies to enhance remyelination have been shown to facilitate functional recovery following SCI (Keirstead et al., 2005; Karimi-Abdolrezaee et al., 2006; Cao et al., 2010). However, these treatments are not effective when applied to chronic injuries (Keirstead et al., 2005; Karimi-Abdolrezaee et al., 2006), casting doubt whether there is a significant population of chronically demyelinated axons to target with myelination strategies. There is some evidence that the chronic application of myelinating cells may improve function since modest improvements in BBB scores and paw positioning were observed in contused rats that received
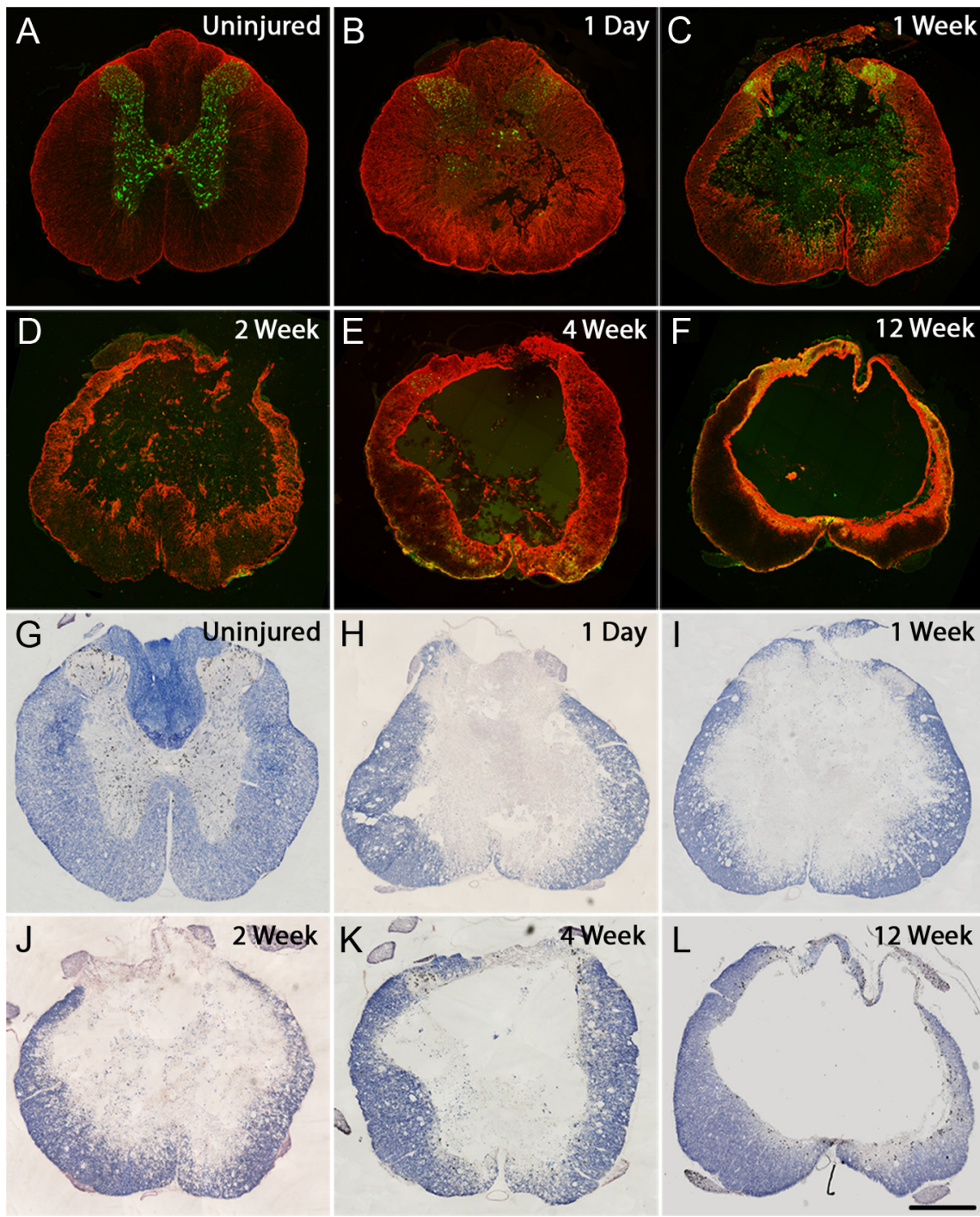

Figure 10. A 150 kdyn contusion leads to progressive cell loss, reactive gliosis, and cavitation. GFAP and NeuN immunohistochemistry $(\boldsymbol{A}-\boldsymbol{F})$ and eriochrome cyanine staining $(\boldsymbol{G}-\boldsymbol{L})$ in transverse sections of the spinal cord through epicenter of the injury demonstrate the pathological changes taking place from acute to chronic time points after injury ( $1 \mathrm{~d}$ to 12 weeks) and can be compared with uninjured spinal cord $(\boldsymbol{A}, \boldsymbol{G})$. GFAP (astrocytes; red) and NeuN (neuronal cell bodies; green) costaining illustrates the progressive destruction of the gray matter and neuronal cell loss that occurs following injury as well as the pronounced increase in reactive gliosis acutely following injury $(\boldsymbol{B})$, which at later stages becomes more localized to the borders of the cavity, forming a dense glial scar $(\boldsymbol{C} \boldsymbol{F})$. Staining with eriochrome cyanine (to demarcate gray and white matter areas) highlights the initial phase of mass necrosis in the spinal parenchyma at early stages $(\boldsymbol{H}-\boldsymbol{J})$ followed by clearance of the debris, resulting in large central cavities surrounded by a spared rim of white matter at chronic postinjury time points $(\boldsymbol{K}, \boldsymbol{L})$. Scale bar, $500 \mu \mathrm{m}$.

Schwann cell transplants 2 months following injury (Barakat et al., 2005), although anatomical evidence suggested that the beneficial effects may have been due to axon growth and/or sprouting rather than remyelination. In the present study, we found that despite extensive remyelination at 12 weeks after injury there remained a small but significant proportion of demyelinated axons. Importantly, we also demonstrate that, even in chronic stages of SCI, conduction can be restored to some axons upon cooling; these are most likely to be the chronically demyelinated axons observed by EM. This further demonstrates the potential of viable but nonconducting axons as important therapeutic targets. Whether such therapies should involve remyelination, or other methods of reducing conduction block, remains for further study.

Interestingly, we also demonstrated that the myelination status of axons varies depending on their location relative to the 

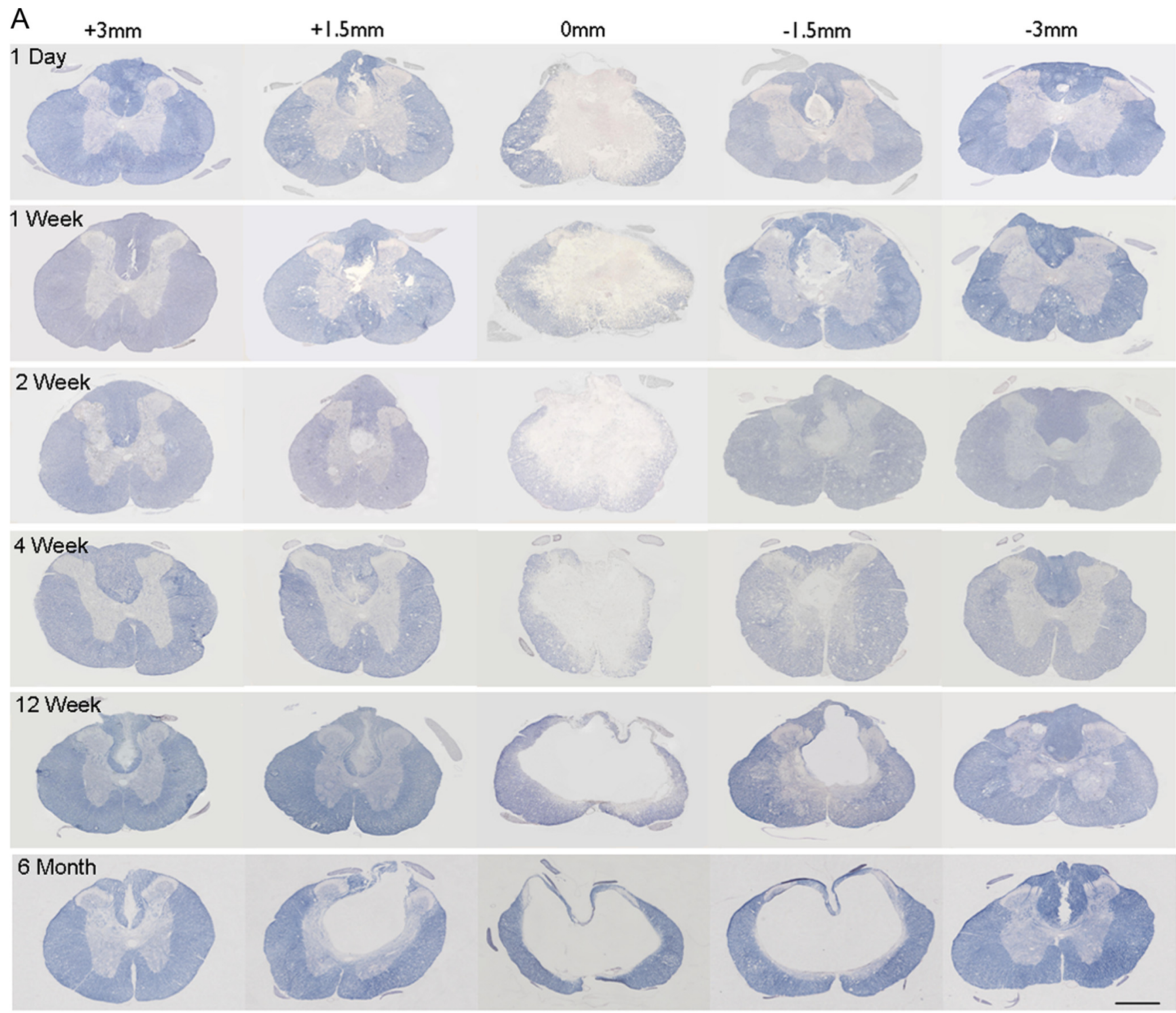

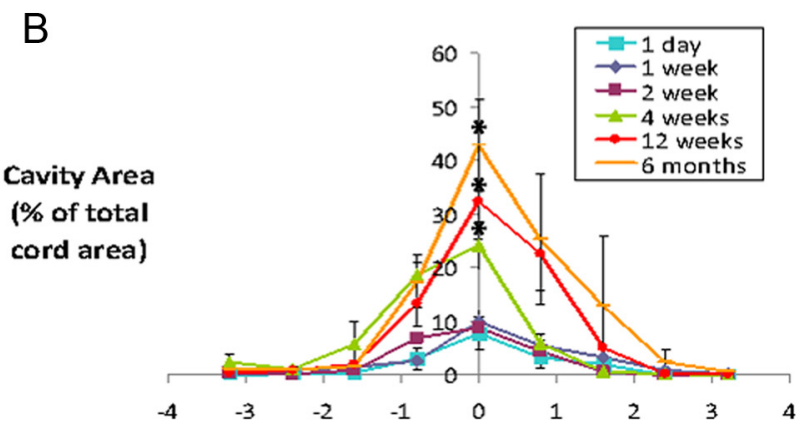

Tissue Depth Relative to Lesion Epicentre (mm)

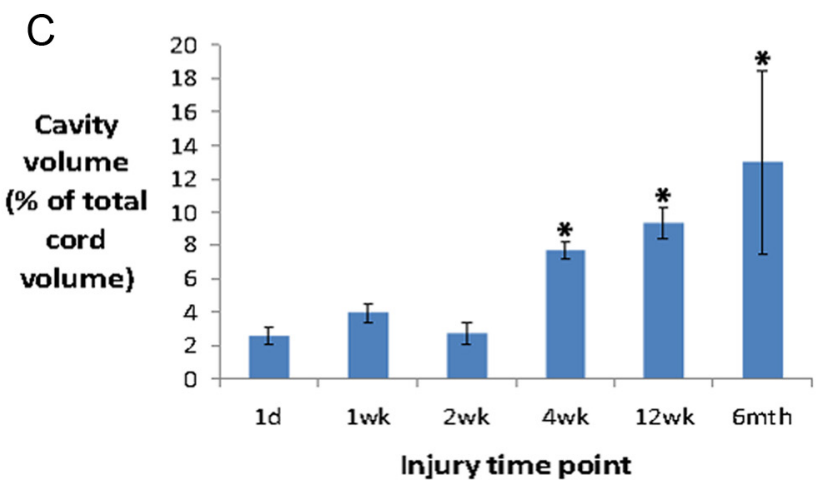

Figure 11. Cavity formation and rostrocaudal degeneration gradually increases over time following spinal contusion. $A$, Eriochrome cyanine staining of serial tissue sections from $3 \mathrm{~mm}$ rostral to $3 \mathrm{~mm}$ caudal to the lesion site ( $0 \mathrm{~mm}$ indicates the lesion epicenter) at a number of postinjury time points shows stereotypical tissue degeneration and progressive cavity formation; mass destruction of both white and gray matter is apparent at the lesion epicenter at all postinjury time points; rostrocaudal degeneration was mainly restricted to the dorsal columns at early postinjury time points, with increasing rostrocaudal cavitation observed at later stages. $B$, Quantification of cavity area (expressed as percentage of spinal cord area) at $800 \mu \mathrm{m}$ intervals through the extent of the injury confirms significantly increased cavity size and rostrocaudal degeneration in the later stages of injury (from 4 weeks). C, Similarly, quantification of total lesion volume, expressed as percentage of total spinal cord volume through the same section of spinal cord shows a significantly increased lesion volume at all chronic injury time points (from 4 weeks onward), compared with the earlier postinjury time points (up to 2 weeks). Error bars represent SEM. The asterisks denote significantly increased cavity area $(<0.05$, two-way RM ANOVA, Bonferroni's post hoc) and cavity volume $(p<$ 0.01, one-way ANOVA, Tukey's post hoc) at all chronic injury time points ( 4 weeks, 12 weeks, and 6 months), compared with acute and subacute injury time points ( $1 \mathrm{~d}, 1$ week, 2 weeks). Scale bar, $500 \mu \mathrm{m}$. 
cavity border, with more demyelinated axons found closer to the cavity border. This has not previously been studied in the rat and highlights the clinical relevance of the injury model since substantial demyelination in the pericavity region surrounded by a rim of subpial myelinated axons has also been observed in chronic human SCIs (Guest et al., 2005). This phenomena may arise because a number of pathological processes that contribute to necrosis of oligodendrocytes, such as vascular responses, ischemia, excitotoxicity, and inflammation (Hagg and Oudega, 2006), are likely to be maximal within and close to the cavity.

We also demonstrated many Schwann cell-associated remyelinated axons in contused spinal cords at chronic postcontusion time points, which is also in line with observations from human SCI studies (Bruce et al., 2000; Guest et al., 2005). These Schwann cells may originate in the peripheral spinal roots and migrate to the spinal cord as a result of injury-induced compromise of the central glial barrier (Franklin and Blakemore, 1993). Alternatively, they may derive from CNS-resident oligodendrocyte precursors (Zawadzka et al., 2010).

\section{Behavioral and anatomical correlates}

Recovery of behavioral function largely corresponded with the electrophysiological data, although correlation studies revealed that the ladder test more closely corresponded to the time course of recovered conduction than the $\mathrm{BBB}$ test, presumably since accurate paw placement and gripping incorporate a sensory element, whereas the BBB scale primarily assesses locomotor function (Basso et al., 1995; Metz and Whishaw, 2002). These data highlight the potential limitations of using BBB scoring alone, since it may not be sensitive enough to bring out subtle changes in behavioral function. While our observations of progressive destruction of white and gray matter tissue and cavity formation are in line with previous studies (Beattie et al., 1997; Rabchevsky et al., 2001), we additionally report here the rapid loss of spinal neurons at the lesion epicenter, evident at only $1 \mathrm{~d}$ after contusion. Although the loss of T10 spinal neurons is unlikely to contribute significantly to hindlimb dysfunction, since they largely innervate trunk musculature, this observation nevertheless provides a useful outcome measure for this injury model. Furthermore, although the phenomenon of glial scarring is well established in the literature (Reier et al., 1983), here we showed the transition from gross and widespread astrogliosis in acute and subacute stages of injury through to the formation of a more condensed glial scar around the cavity borders at the injury epicenter. Areas dense in glial scar are particularly hostile to growth (Fawcett and Asher, 1999; Fitch and Silver, 2008), and, interestingly, these GFAP-enriched areas are exactly where we observed the most extensive demyelination.

Thus, we provide robust evidence of chronic conduction failure in spared axons following spinal contusion injury and have identified a population of viable, but nonconducting axons. Furthermore, we show that these axons remain accessible to strategies to restore conduction, even at chronic stages of a SCI, and thus are a prime therapeutic target.

\section{References}

Alilain WJ, Horn KP, Hu H, Dick TE, Silver J (2011) Functional regeneration of respiratory pathways after spinal cord injury. Nature 475:196-200.

Alto LT, Havton LA, Conner JM, Hollis Ii ER, Blesch A, Tuszynski MH (2009) Chemotropic guidance facilitates axonal regeneration and synapse formation after spinal cord injury. Nat Neurosci 12:1106-1113.

Atkinson PP, Atkinson JL (1996) Spinal shock. Mayo Clin Proc 71:384-389.

Barakat DJ, Gaglani SM, Neravetla SR, Sanchez AR, Andrade CM, Pressman Y, Puzis R, Garg MS, Bunge MB, Pearse DD (2005) Survival, integration, and axon growth support of glia transplanted into the chronically contused spinal cord. Cell Transplant 14:225-240.

Basso DM, Beattie MS, Bresnahan JC (1995) A sensitive and reliable locomotor rating scale for open field testing in rats. J Neurotrauma 12:1-21.

Beattie MS, Bresnahan JC, Komon J, Tovar CA, Van Meter M, Anderson DK, Faden AI, Hsu CY, Noble LJ, Salzman S, Young W (1997) Endogenous repair after spinal cord contusion injuries in the rat. Exp Neurol 148:453-463.

Beaumont E, Onifer SM, Reed WR, Magnuson DS (2006) Magnetically evoked inter-enlargement response: an assessment of ascending propriospinal fibers following spinal cord injury. Exp Neurol 201:428-440.

Blakemore WF, Murray JA (1981) Quantitative examination of internodal length of remyelinated nerve fibres in the central nervous system. J Neurol Sci 49:273-284.

Blight AR (1983) Axonal physiology of chronic spinal cord injury in the cat: intracellular recording in vitro. Neuroscience 10:1471-1486.

Bostock H, Sherratt RM, Sears TA (1978) Overcoming conduction failure in demyelinated nerve fibres by prolonging action potentials. Nature 274:385-387.

Bradbury EJ, Carter LM (2011) Manipulating the glial scar: chondroitinase $A B C$ as a therapy for spinal cord injury. Brain Res Bull 84:306-316.

Bradbury EJ, Moon LD, Popat RJ, King VR, Bennett GS, Patel PN, Fawcett JW, McMahon SB (2002) Chondroitinase ABC promotes functional recovery after spinal cord injury. Nature 416:636-640.

Bruce JH, Norenberg MD, Kraydieh S, Puckett W, Marcillo A, Dietrich D (2000) Schwannosis: role of gliosis and proteoglycan in human spinal cord injury. J Neurotrauma 17:781-788.

Bunge RP, Puckett WR, Becerra JL, Marcillo A, Quencer RM (1993) Observations on the pathology of human spinal cord injury. A review and classification of 22 new cases with details from a case of chronic cord compression with extensive focal demyelination. Adv Neurol 59:75-89.

Cafferty WB, Duffy P, Huebner E, Strittmatter SM (2010) MAG and OMgp synergize with Nogo-A to restrict axonal growth and neurological recovery after spinal cord trauma. J Neurosci 30:6825-6837.

Cao Q, Zhang YP, Iannotti C, DeVries WH, Xu XM, Shields CB, Whittemore SR (2005) Functional and electrophysiological changes after graded traumatic spinal cord injury in adult rat. Exp Neurol 191 [Suppl 1]:S3-S16.

Cao Q, He Q, Wang Y, Cheng X, Howard RM, Zhang Y, DeVries WH, Shields CB, Magnuson DS, Xu XM, Kim DH, Whittemore SR (2010) Transplantation of ciliary neurotrophic factor-expressing adult oligodendrocyte precursor cells promotes remyelination and functional recovery after spinal cord injury. J Neurosci 30:2989-3001.

Dumont RJ, Okonkwo DO, Verma S, Hurlbert RJ, Boulos PT, Ellegala DB, Dumont AS (2001) Acute spinal cord injury, Part I: Pathophysiologic mechanisms. Clin Neuropharmacol 24:254-264.

Ellingson BM, Kurpad SN, Schmit BD (2008) Characteristics of mid- to long-latency spinal somatosensory evoked potentials following spinal trauma in the rat. J Neurotrauma 25:1323-1334.

Fawcett JW, Asher RA (1999) The glial scar and central nervous system repair. Brain Res Bull 49:377-391.

Fitch MT, Silver J (2008) CNS injury, glial scars, and inflammation: Inhibitory extracellular matrices and regeneration failure. Exp Neurol 209:294-301.

Franklin RJ, Blakemore WF (1993) Requirements for Schwann cell migration within CNS environments: a viewpoint. Int J Dev Neurosci 11:641-649.

Guest JD, Hiester ED, Bunge RP (2005) Demyelination and Schwann cell responses adjacent to injury epicenter cavities following chronic human spinal cord injury. Exp Neurol 192:384-393.

Hagg T, Oudega M (2006) Degenerative and spontaneous regenerative processes after spinal cord injury. J Neurotrauma 23:264-280.

Hains BC, Saab CY, Lo AC, Waxman SG (2004) Sodium channel blockade with phenytoin protects spinal cord axons, enhances axonal conduction, and improves functional motor recovery after contusion SCI. Exp Neurol 188:365-377.

Hellal F, Hurtado A, Ruschel J, Flynn KC, Laskowski CJ, Umlauf M, Kapitein LC, Strikis D, Lemmon V, Bixby J, Hoogenraad CC, Bradke F (2011) Microtubule stabilization reduces scarring and causes axon regeneration after spinal cord injury. Science 331:928-931.

Hubscher CH, Johnson RD (2006) Chronic spinal cord injury induced changes in the responses of thalamic neurons. Exp Neurol 197:177-188. 
Hunanyan AS, García-Alías G, Alessi V, Levine JM, Fawcett JW, Mendell LM, Arvanian VL (2010) Role of chondroitin sulfate proteoglycans in axonal conduction in mammalian spinal cord. J Neurosci 30:7761-7769.

Iaci JF, Vecchione AM, Zimber MP, Caggiano AO (2007) Chondroitin sulfate proteoglycans in spinal cord contusion injury and the effects of chondroitinase treatment. J Neurotrauma 24:1743-1759.

Jones LL, Margolis RU, Tuszynski MH (2003) The chondroitin sulfate proteoglycans neurocan, brevican, phosphacan, and versican are differentially regulated following spinal cord injury. Exp Neurol 182:399-411.

Kakulas BA (1999) A review of the neuropathology of human spinal cord injury with emphasis on special features. J Spinal Cord Med 22:119-124.

Karimi-Abdolrezaee S, Eftekharpour E, Wang J, Morshead CM, Fehlings MG (2006) Delayed transplantation of adult neural precursor cells promotes remyelination and functional neurological recovery after spinal cord injury. J Neurosci 26:3377-3389.

Keirstead HS, Nistor G, Bernal G, Totoiu M, Cloutier F, Sharp K, Steward O (2005) Human embryonic stem cell-derived oligodendrocyte progenitor cell transplants remyelinate and restore locomotion after spinal cord injury. J Neurosci 25:4694-4705.

Khan T, Havey RM, Sayers ST, Patwardhan A, King WW (1999) Animal models of spinal cord contusion injuries. Lab Anim Sci 49:161-172.

Lasiene J, Shupe L, Perlmutter S, Horner P (2008) No evidence for chronic demyelination in spared axons after spinal cord injury in a mouse. J Neurosci 28:3887-3896.

McDonald WI, Sears TA (1969) Effect of demyelination on conduction in the central nervous system. Nature 221:182-183.

Metz GA, Whishaw IQ (2002) Cortical and subcortical lesions impair skilled walking in the ladder rung walking test: a new task to evaluate foreand hindlimb stepping, placing, and co-ordination. J Neurosci Methods 115:169-179.

Metz GA, Curt A, van de Meent H, Klusman I, Schwab ME, Dietz V (2000) Validation of the weight-drop contusion model in rats: a comparative study of human spinal cord injury. J Neurotrauma 17:1-17.

Nashmi R, Fehlings MG (2001) Changes in axonal physiology and morphology after chronic compressive injury of the rat thoracic spinal cord. Neuroscience 104:235-251.

Nashmi R, Imamura H, Tator CH, Fehlings MG (1997) Serial recording of somatosensory and myoelectric motor evoked potentials: role in assessing functional recovery after graded spinal cord injury in the rat. J Neurotrauma 14:151-159.

Nashmi R, Jones OT, Fehlings MG (2000) Abnormal axonal physiology is associated with altered expression and distribution of Kv1.1 and Kv1.2 $\mathrm{K}^{+}$channels after chronic spinal cord injury. Eur J Neurosci 12:491-506.

Norenberg MD, Smith J, Marcillo A (2004) The pathology of human spinal cord injury: defining the problems. J Neurotrauma 21:429-440.

Onifer SM, Nunn CD, Decker JA, Payne BN, Wagoner MR, Puckett AH, Massey JM, Armstrong J, Kaddumi EG, Fentress KG, Wells MJ, West RM, Calloway CC, Schnell JT, Whitaker CM, Burke DA, Hubscher CH (2007) Loss and spontaneous recovery of forelimb evoked potentials in both the adult rat cuneate nucleus and somatosensory cortex following contusive cervical spinal cord injury. Exp Neurol 207:238-247.
Rabchevsky AG, Fugaccia I, Sullivan PG, Scheff SW (2001) Cyclosporin A treatment following spinal cord injury to the rat: behavioral effects and stereological assessment of tissue sparing. J Neurotrauma 18:513-522.

Rasminsky M (1973) The effects of temperature on conduction in demyelinated single nerve fibers. Arch Neurol 28:287-292.

Reier PJ, Stensaas LJ, Guth L (1983) The astrocytic scar as an impediment to regeneration in the central nervous system. In: Spinal cord reconstruction (Kao CC, Bunge RP, Reier PJ, eds), pp 163-195. New York: Raven.

Salazar DL, Uchida N, Hamers FP, Cummings BJ, Anderson AJ (2010) Human neural stem cells differentiate and promote locomotor recovery in an early chronic spinal cord injury NOD-scid mouse model. PLoS One 5:e12272.

Scheff SW, Rabchevsky AG, Fugaccia I, Main JA, Lumpp JE Jr (2003) Experimental modeling of spinal cord injury: characterization of a forcedefined injury device. J Neurotrauma 20:179-193.

Sharma HS, Westman J, Olsson Y, Alm P (1996) Involvement of nitric oxide in acute spinal cord injury: an immunocytochemical study using light and electron microscopy in the rat. Neurosci Res 24:373-384.

Shuman SL, Bresnahan JC, Beattie MS (1997) Apoptosis of microglia and oligodendrocytes after spinal cord contusion in rats. J Neurosci Res 50:798-808.

Smith KJ, Felts PA, John GR (2000) Effects of 4-aminopyridine on demyelinated axons, synapses and muscle tension. Brain 123:171-184.

Smith PM, Jeffery ND (2005) Spinal shock-comparative aspects and clinical relevance. J Vet Intern Med 19:788-793.

Springer JE, Rao RR, Lim HR, Cho SI, Moon GJ, Lee HY, Park EJ, Noh JS, Gwag BJ (2010) The functional and neuroprotective actions of Neu2000, a dual-acting pharmacological agent, in the treatment of acute spinal cord injury. J Neurotrauma 27:139-149.

Tang X, Davies JE, Davies SJ (2003) Changes in distribution, cell associations, and protein expression levels of NG2, neurocan, phosphacan, brevican, versican $\mathrm{V} 2$, and tenascin-C during acute to chronic maturation of spinal cord scar tissue. J Neurosci Res 71:427-444.

Tator CH, Fehlings MG (1991) Review of the secondary injury theory of acute spinal cord trauma with emphasis on vascular mechanisms. J Neurosurg 75:15-26.

Totoiu MO, Keirstead HS (2005) Spinal cord injury is accompanied by chronic progressive demyelination. J Comp Neurol 486:373-383.

Waxman SG (1989) Demyelination in spinal cord injury. J Neurol Sci 91:1-14.

Winkler T, Sharma HS, Stålberg E, Badgaiyan RD, Alm P, Westman J (1998) Spinal cord evoked potentials and edema in the pathophysiology of rat spinal cord injury. Involvement of nitric oxide. Amino Acids 14:131-139.

Zawadzka M, Rivers LE, Fancy SP, Zhao C, Tripathi R, Jamen F, Young K, Goncharevich A, Pohl H, Rizzi M, Rowitch DH, Kessaris N, Suter U, Richardson WD, Franklin RJ (2010) CNS-resident glial progenitor/ stem cells produce Schwann cells as well as oligodendrocytes during repair of CNS demyelination. Cell Stem Cell 6:578-590.

Zorner B, Schwab ME (2010) Anti-Nogo on the go: from animal models to a clinical trial. Ann N Y Acad Sci 1198 [Suppl 1]:E22-E34. 